\title{
Tree Representations via Ordinal Machines
}

\author{
Philipp Schlicht \\ Mathematical Institute, University of Bonn \\ Endenicher Allee 60, 53115 Bonn, Germany \\ schlicht@math.uni-bonn. de \\ Benjamin Seyfferth \\ Mathematical Institute, University of Bonn \\ Endenicher Allee 60, 53115 Bonn, Germany \\ seyfferthemath. uni-bonn. de
}

\begin{abstract}
We study sets of reals computable by ordinal Turing machines with a tape of length the ordinals that are steered by a standard Turing program. The machines halt at some ordinal time or diverge. We construct tree representations for ordinal semidecidable sets of reals from ordinal computations. The aim is to generalize uniformization results to classes of sets which are semi-decidable by computations with input-dependent bounds on the halting time. We further briefly examine the jump structure and nondeterminism.
\end{abstract}

Keywords: ordinal machines, tree representations, uniformization, nondeterministic ordinal computations

\section{Introduction}

Ordinal computability studies generalized computability theory by means of classical machine models that operate on ordinals instead of natural numbers. Starting with Joel Hamkins' and Andy Lewis' Infinite Time Turing Machines (ITTM) [3], recent years have seen several of those models which provided alternate approaches and new aspects for various ideas from logic, set theory and classical areas of generalized computability theory. With ITTMs, the machine may carry out a transfinite ordinal number of steps while writing $0 \mathrm{~s}$ and $1 \mathrm{~s}$ on tapes of length $\omega$. This is achieved by the addition of a limit rule that governs the behavior of the machine at limit times. The $0 \mathrm{~s}$ and $1 \mathrm{~s}$ on the $\omega$-long tape are interpreted as subsets of $\omega$ (reals). It turns out that the sets of reals semi-decidable by these machines form a subset of $\Delta_{2}^{1}$. Similar studies have been carried out for infinite time register machines (ITRMs), whose computable reals are exactly the reals in $L_{\omega_{\omega}^{c K}}[8]$.

Another direction of ordinal computability lifts classical computability to study not the subsets of $\omega$, but of an arbitrary ordinal $\alpha$, or even the class Ord of all ordinals. In this case, both space and time are set to that ordinal $\alpha$, i.e. in the Turing context, we deal with machines that utilize a tape of length $\alpha$ and either stop in less than $\alpha$ many steps or diverge. The computation is steered by a standard Turing program and a finite number of ordinal parameters less than $\alpha$ so the machines can talk about ordinals below $\alpha$ in much the same fashion as classical Turing machines can about natural numbers. This approach unveils strong connections to Gödels universe of constructible sets and the classical work on $\alpha$-recursion theory [9].

In the present paper, we aim between these two approaches by analyzing the computable sets of reals of Turing machines with Ord space and time but without allowing arbitrary ordinal parameters. Omission of the parameters leads to a model in which all computational information is contained in the real input, the finite Turing program and the limit rules. We work with ordinal Turing machines (OTMs), the machine model introduced in [7]. Let us briefly review the basic features, for more detail and background the reader is referred to the original paper.

An OTM uses the binary alphabet on a one-sided infinite tape whose cells are indexed by ordinal numbers. At any ordinal point in time, the machine head is located on one of these cells and the machine is in one of finitely many machine states indexed by natural numbers. Since we utilize both Ord space and time, there is no need to use multiple tapes in our definition; any fixed finite number of tapes can be simulated by interleaving the tapes into one. 
A typical program instruction has the form $\left(c, s, c^{\prime}, s^{\prime}, d\right) \in\{0,1\} \times \omega \times\{0,1\} \times \omega \times\{-1,1\}$ and is interpreted as the instruction "If the symbol currently read by the machine's read-write head is $c$ and the machine is currently in state $s$, then overwrite $c$ with the symbol $c^{\prime}$, change the machine state to $s^{\prime}$, and move the head according to $d$ either to the left or to the right". At successor times in the course of the computation, the machine behaves like a standard Turing machine, with the following exception: If the machine head rests on a cell indexed by a limit ordinal or 0 and a "move left"-instruction is carried out, then the head is set to position 0 . The machine accesses the transfinite by the following rule, known as the lim inf-rule:

At a limit time $\lambda$, the machine state is set to the lim inf of the states of previous time, i.e., the least state that was assumed cofinally often in the previous steps. Similarly, we set the tape content for each cell individually to the lim inf of the previous cell contents; in other words, a cell contains a 0 at time $\lambda$ if it contained a 0 cofinally often before $\lambda$, and it contains a 1 at time $\lambda$ otherwise. We also set the head position to the cell indexed by the lim inf over the indices of the cells visited at previous steps in which the machine's state matched the limit stage.

These ordinal machines may be used to describe sets of reals. In order to input a real number into an ordinal Turing machine, we start the computation with an initial tape content coding the real; so the initial tape contents is a sequence of the numbers 0 and 1 written in the cells with finite index. Note that our basic definitions do not involve ordinal parameters as in [7, Definition 2.5], hence our main results refer to pointclasses defined without parameters. Since elements of ${ }^{\omega} \omega$ can be coded in ${ }^{\omega} 2$ via Gödel pairing, we can have elements of ${ }^{\omega} \omega$ as input as well. Thus we will also refer to elements of ${ }^{\omega} \omega$ as real numbers. Let us denote the OTM computation by a program $P$ on input $x$ as $P(x)$ and abbreviate the statement " $P(x)$ halts" as $P(x) \downarrow$. The notions of input and output of an OTM computations are defined as in [7]. We will say that a partial function $f: X \rightarrow Y$ is OTM computable if there is a program $P$ that halts on input $x \in \operatorname{dom} f$ with output $f(x) \in Y$, given a suitable coding of elements of $X$ and $Y$ into OTM tapes.

Definition 1.1. A set of reals $A \subseteq{ }^{\omega} \omega$ is called OTM semi-decidable if there is an ordinal Turing machine that halts if and only if the initial tape content was an element of $A$. $A$ is called OTM decidable if both $A$ and ${ }^{\omega} \omega \backslash A$ are OTM semi-decidable.

Our motivation is to use ordinal machines to refine uniformization results in descriptive set theory. In [2] it is shown that many results in descriptive set theory have simple proofs using admissible sets; we go further than [2] in providing explicit algorithms for the constructions.

In section 2, we shall define an algorithm for searching for infinite branches in the Shoenfield tree. This implies that the $\Sigma_{2}^{1}$ sets of reals are exactly the OTM semi-decidable sets of reals. As a consequence, we will re-establish Shoenfield's absoluteness theorem from the perspective of ordinal computability. The fact that the $\Sigma_{2}^{1}$ sets of reals are exactly the OTM semi-decidable sets of reals may be alternatively obtained from $\Sigma_{2}^{1}$ absoluteness and the fact that bounded truth in $L$ is an OTM computable relation (for the latter see [7]).

In section 3, we shall introduce a tree representation for $\Sigma_{2}^{1}$ sets that is based on finite fragments of OTM computations. We will apply the algorithm in section 2 to this tree representation to obtain our main result: Uniformization for classes of sets OTM semi-decidable by computations with input-dependent upper bounds on the halting time.

Section 4 introduces a notion of nondeterministic OTM computations and establishes that nondeterministically OTM decidable sets are already deterministically so. We shall then show that the jump structure of our machines depends on set theoretic assumptions.

\section{Computing the Shoenfield Tree}

In this section, we define an OTM algorithm searching for branches in the Shoenfield tree. To assist in the computations, let us fix the following OTM computable functions. The Gödel pairing function is a bijection $\langle\cdot, \cdot\rangle:$ Ord $\times$ Ord $\rightarrow$ Ord. Elements of Baire space can be represented as subsets of $\omega$ by coding their graph via Gödel pairing. The function $o: \omega \rightarrow<\omega \omega$ is a computable bijection providing a computable enumeration of the basic open sets $O(i)$ of the Baire space ${ }^{\omega} \omega$, where $O(i)$ denotes the basic open set defined by the sequence $o(i)$. Let us recall some basic notation commonly used in classical descriptive set theory: If $T$ is a tree on ${ }^{k} \omega \times \alpha$ and $x \in{ }^{k}\left({ }^{\omega} \omega\right)$, let $T_{x}=\left\{u \in{ }^{n} \alpha:(x\lceil n, u) \in T, n \in \omega\}\right.$. If $s \in{ }^{k}\left({ }^{m} \omega\right)$, let $T_{s}=\left\{u \in{ }^{n} \alpha:(s\lceil n, u) \in T, n<m\}\right.$. 
We will make use of the standard tree representation for $\Pi_{1}^{1}$ sets due to Luzin and Sierpiński. Recall that a set $B \subseteq{ }^{k}\left({ }^{\omega} \omega\right)$ is $\Pi_{1}^{1}$ if there is a tree $T$ on ${ }^{k} \omega \times \omega$ such that the relation $\left\{(x, i) \mid o(i) \in T_{x}\right\}$ is computable and $x \in B$ if and only if $T_{x}$ is well-founded. Let us call $T$ the Luzin-Sierpinski tree for $B$. The tree $T_{x}$ is well-founded if and only if there is an order-preserving embedding of $T_{x}$ into some countable ordinal $\alpha$. Hence, to check whether $x \in B$, we can look for a suitable infinite branch in the tree $S$ on ${ }^{k} \omega \times \omega_{1}$ of all pairs $(s, u)$ with $s \in{ }^{k}\left({ }^{n} \omega\right)$ and $u \in{ }^{n} \omega_{1}$ for some $n \in \omega$ where $u$ codes an order-preserving map $f_{u}: T_{s} \cap\{o(i) \mid i<\operatorname{length}(u)\} \rightarrow \omega_{1}$. This is the Shoenfield tree projecting to $B$.

Let us first define an algorithm searching the Shoenfield tree for a $\Pi_{1}^{1}$ set $B \subseteq{ }^{\omega} \omega$. Let $T$ be the Luzin-Sierpiński tree for $B$. We would like the algorithm to halt on input $x \in{ }^{\omega} \omega$ if and only if $x \in B$. Depth-first-search (DFS) is employed to find an infinite branch in the subtree of $S$ that consists of the pairs $(s, u)$, where $s=x\lceil n$ for some $n \in \omega$. In other words, we will search $S_{x}$, which is a tree on $\omega_{1}$. Clearly, membership in $S$ of any given pair $(s, u)$ is OTM decidable inside every admissible set, as the property $o(i) \in T_{s}$ is computable in the classical sense. Note that $\omega$ may be used as a constant, since the constant function with value $\omega$ is OTM computable.

\section{Algorithm 2.1.}

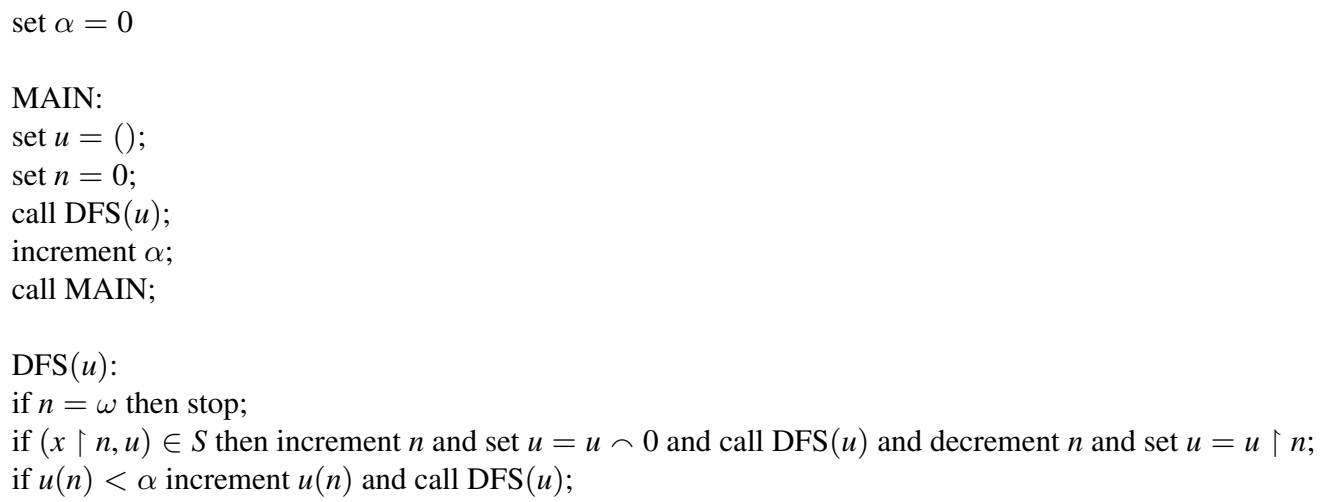

The algorithm starts with the empty sequence $u=()$ in stage $\alpha=0$. Whenever $\operatorname{DFS}(u)$ is called, all possible extensions of $u$ by a single ordinal $\beta<\alpha$ are considered. When all $\beta<\alpha$ have been tried, DFS $(u)$ ends. If a extension $u \frown \beta \in S_{x}$ is found, the recursion will immediately try to extend it further and $\operatorname{DFS}(u \frown \beta)$ is called. Whenever the algorithm tries an extension $u \frown \beta$ that is not in $S_{x}$, this extension is not followed further and $u \frown$ $(\beta+1)$ is tried next. If the length $n$ of $u$ has reached $\omega$, a branch is found, i.e., $u$ codes an order preserving embedding of $T_{x}$ into the ordinal $\alpha$. If no branch can be found, the recursion eventually breaks down, $\alpha$ is incremented, and the algorithm starts over with the empty sequence.

Throughout the algorithm, the variable $u$ is stored in an extra tape whose $n$-th cell contains a 1 if and only if $n=\langle p, q\rangle$ and $u(p) \geq q$ and 0 otherwise. Therefore, the variable also contains the desired value at limit times.

Lemma 2.2. The algorithm will find the lexicographically least infinite branch through $S_{x}$, if there is one.

Proof. It is clear that if the algorithm finds a branch, it will find the lexicographically least. So we have to show that this branch is eventually found. Let $v \in{ }^{\omega} \omega_{1}$ be the lexicographically least branch of $S_{x}$ and let $\gamma$ be the supremum of the ordinals in $v$. The tree $S_{x} \cap{ }^{<\omega} \gamma$ is countable. Observe that the algorithm visits exactly the nodes of $S_{x} \cap{ }^{<\omega} \gamma$ in the stages $\alpha<\gamma$ and that every node is visited only once. Since this subtree contains no infinite branches, the algorithm sets $\alpha=\gamma$ after countably many steps. Note that in stage $\gamma$, the algorithm will first visit the countably many sequences $w \in S_{x}$ that are lexicographically smaller than $v\lceil$ length $(w)$. No node $w$ that is lexicographically greater than $v \uparrow$ length $(w)$ is visited before the algorithm examines every initial segment of $v$, so the algorithm eventually finds the branch in countable time.

Now consider a $\Sigma_{2}^{1}$ set $A \subseteq{ }^{\omega} \omega$ and a $\Pi_{1}^{1}$ set $B \subseteq{ }^{\omega} \omega \times{ }^{\omega} \omega$ such that $p(B)=A$. We will modify the previous algorithm to semi-decide the set $A$. Let $T \subseteq\left({ }^{2} \omega \times \omega\right)^{<\omega}$ be the Luzin-Sierpiński tree for $B$. The Shoenfield tree $S$ 
for $B$ is the tree of all $(s, t, u)$ where $u$ codes an order-preserving embedding $f_{u}: T_{s, t} \rightarrow$ Ord. Since $B=p([S])$ and $A=p(B)$, we have $x \in A$ if and only if the tree $S_{x}$ (on $\omega \times \omega_{1}$ ) has an infinite branch. In order to find such a branch for a given $x$, the algorithm proceeds in stages $\alpha \in$ Ord. In each stage $\alpha$, depth-first-search is employed to find an infinite branch in the subtree of $S_{x}$ which consists of the pairs $(t, u)$ where $u$ is a tuple of ordinals below $\alpha$.

\section{Algorithm 2.3.}

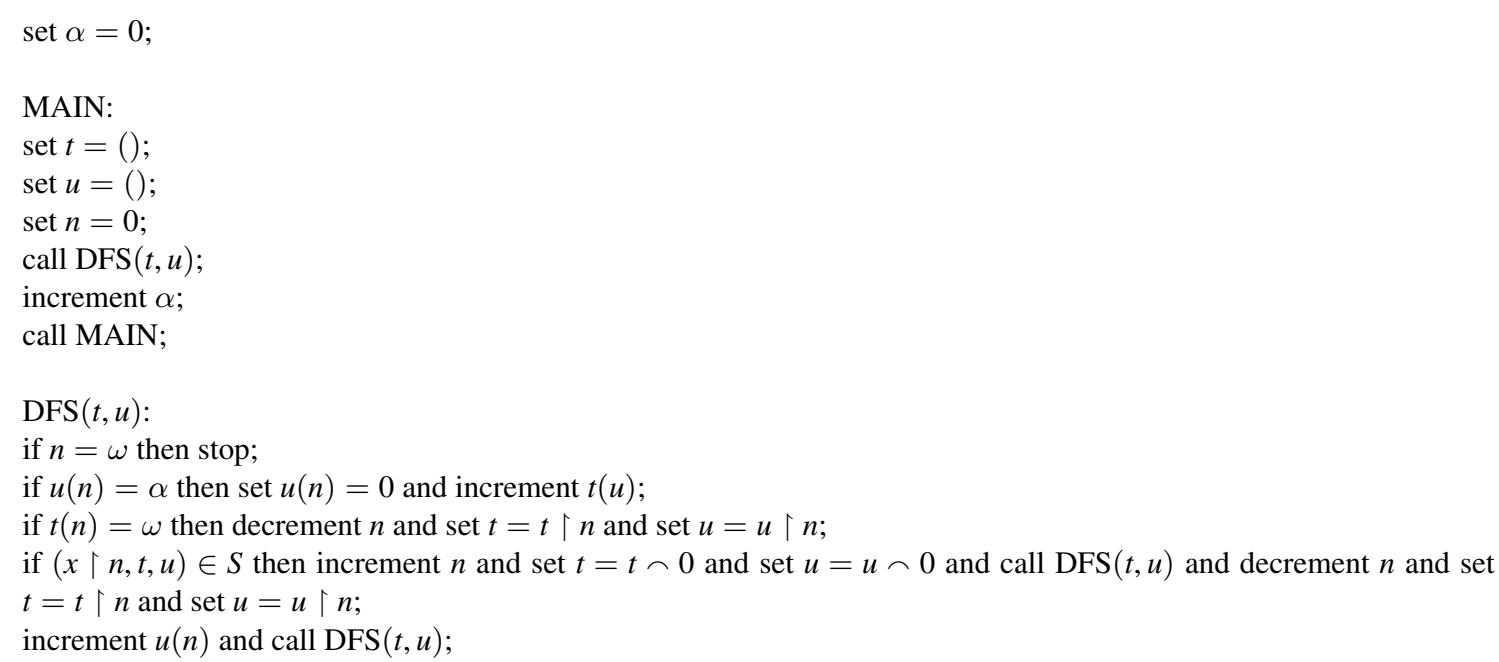

Here in every call of $\operatorname{DFS}(t, u)$, the algorithm tries to extend $t$ and $u$ simultaneously by all pairs $(m, \beta)$ with $m \in \omega$ and $\beta<\alpha$. Again, if $(t \frown m, u \frown \beta) \in S_{x}$, the sequence is immediately extended further and $\operatorname{DFS}(t \frown m, u \frown \beta)$ is called. Otherwise, $(t \frown m, u \frown \beta+1)$ is tried next. If for all $\beta<\alpha(t \frown m, u \frown \beta)$ cannot be extended further, then $(t \frown m+1, u \frown 0)$ is tried next, and so on.

Lemma 2.4. The algorithm will find the lexicographically least $z$ such that $S_{x, z}$ has a branch, and the lexicographically least branch $v$ through $S_{x, z}$, if such a real z exists.

Proof. Assume $z$ and $v$ are as required. As in Lemma 2.2, we can see that before stage $\gamma$ (where $\gamma$ is the supremum of the range of the embedding coded by $v$ ), only countably many nodes are visited. In stage $\gamma$, only countably many nodes are visited before the branch $(z, v)$ is found.

It is straightforward to generalize this algorithm to semi-decide $\Sigma_{2}^{1}$ subsets of ${ }^{k}\left({ }^{\omega} \omega\right)$.

Remark 2.5. Notice that the halting time of any halting OTM computation with input a real $x$ is countable: If we collapse a countable elementary substructure of some $L_{\alpha}[x]$ which contains the computation as an element, the collapsing function maps the computation to an initial segment, since OTM computations are absolute between transitive models of KP (see [7, Lemma 2.6]). So the computation in fact halts at a countable time.

Proposition 2.6. The OTM semi-decidable subsets of ${ }^{k}\left({ }^{\omega} \omega\right)$ are exactly the $\Sigma_{2}^{1}$ sets. The OTM decidable sets are the $\Delta_{2}^{1}$ sets.

Proof. The Shoenfield tree and Lemma 2.4 prove that all $\Sigma_{2}^{1}$ sets are OTM semi-decidable. On the other hand, OTM semi-decidable sets are easily seen to be $\Sigma_{2}^{1}$ definable. The second statement follows.

If one wants to prove Shoenfield absoluteness without referring to the Shoenfield tree (which is defined in terms of descriptive set theory), it can be replaced with a tree defined by only computational means. We will describe such a tree in Remark 3.6. It also can replace the Shoenfield tree in all following proofs.

From the algorithms, we obtain short proofs of several results in classical descriptive set theory (cf. [5, 6]). 
Corollary 2.7. Suppose $M$ is a transitive model of $\mathrm{KP}$ with $\omega_{1} \subseteq M$. Then $\Sigma_{2}^{1}$ relations are absolute between $M$ and $V$.

Proof. Since OTM computations are absolute between transitive models of KP, so is membership in $\Sigma_{2}^{1}$ sets.

Corollary 2.8. Every $\Sigma_{2}^{1}$ binary relation on the reals has a $\Sigma_{2}^{1}$ uniformization and every $\Pi_{1}^{1}$ binary relation on the reals has a $\Pi_{1}^{1}$ uniformization.

Proof. Suppose $A \subseteq{ }^{\omega} \omega \times{ }^{\omega} \omega$ is a $\Sigma_{2}^{1}$ set. The algorithm semi-deciding $(x, y) \in A$ can be modified to search for a $y$ given $x$ as input. As we added the search for sequences $t \in{ }^{<\omega} \omega$ to Algorithm 2.1 to obtain Algorithm 2.3, we may also add another search for $s \in<\omega \omega$ with $(s, t, u) \in S_{x}$. An argument analogous to Lemmas 2.2 and 2.4 proves that the lexicographically least branch $(y, z, v)$ through $S_{x}$ is found. This corresponds to the lexicographically least branch through $S_{x, y}$, therefore $(x, y) \in A$. For any $\Pi_{1}^{1}$ binary relation, a similar modification of Algorithm 2.1 yields an algorithm semi-deciding a uniformization such that for any pair $(x, y)$ in the uniformizing function, the algorithm halts before the least $(x, y)$-admissible ordinal $\omega_{1}^{x, y}$ above $\omega$. Hence the uniformization is $\Pi_{1}^{1}$ by the Spector-Gandy theorem.

This immediately implies:

Corollary 2.9. Every nonempty $\Sigma_{2}^{1}$ set of reals has a $\Sigma_{2}^{1}$ member, i.e. some $x$ such that $\{x\}$ is a $\Sigma_{2}^{1}$ set, and every nonempty $\Pi_{1}^{1}$ set of reals has a $\Pi_{1}^{1}$ member.

Remark 2.10. The proof of Corollary 2.8 shows that any function from the reals to the reals with OTM semidecidable graph is OTM computable. This is false in general, e.g. when we consider OTM programs $P$ such that $P(x)$ halts before $\omega_{1}^{x}$ for all $x$ with $P(x) \downarrow$. Let us consider a $\Pi_{1}^{1}$ function $f$, obtained via $\Pi_{1}^{1}$ uniformization, mapping a real $x$ to a code for a wellfounded countable model containing $x$ of the theory $T$, where $T$ is the extension of KP requiring that there is an admissible ordinal. Although its graph is semi-decidable by such a program, it is easy to see that $f$ is not OTM computable by a program of this type.

Corollary 2.11. Every $\Sigma_{2}^{1}$ set is the union of $\omega_{1}$ many Borel sets.

Proof. Given a $\Sigma_{2}^{1}$ set $A$, let $P$ be an OTM which terminates on input $x$ if and only if $x \in A$. Let $A_{\beta}$ denote the set of reals $x$ such that $P(x)$ terminates before stage $\beta$. Then $A$ is the union of the sets $A_{\beta}$. To see that each $A_{\beta}$ is Borel, let $a_{\beta}$ be a real coding the supremum $\gamma_{\beta}$ over the halting times of the algorithm if restricted to at most $\beta$ stages. ${ }^{1}$ Then a real $x$ is an element of $A_{\beta}$ if and only if for some (for every) real $c$ coding a computation along $a_{\beta}$, this computation halts. This shows that $A_{\beta}$ is $\Delta_{1}^{1}$ and hence Borel by Suslin's theorem.

Corollary 2.12. Every $\Sigma_{2}^{1}$ set has a $\Sigma_{2}^{1}$ norm.

Proof. Let $A$ be a $\Sigma_{2}^{1}$ set and let $P$ be an algorithm semi-deciding $A$. The desired norm is given by the map $\phi$ where $P$ halts at time $\phi(x)$ on input $x$. Let $x \leq y(x<y)$ if $P(x)$ halts (strictly) before $P(y)$, or $P(x)$ halts and $P(y)$ does not halt. Then $y \in A$ and $x \leq y$ imply $x \in A$. Using the algorithm, it is easy to see that the relations $\leq$ and $<$ are OTM semi-decidable, hence $\Sigma_{2}^{1}$. We can define a $\Pi_{2}^{1}$ relation $\leq^{\prime}$ by $x \leq^{\prime} y \leftrightarrow \neg y<x$ which coincides with $\leq$ on $A$ and again $y \in A$ and $x \leq^{\prime} y$ implies $x \in A$. Hence $\phi$ is a $\Sigma_{2}^{1}$ norm on $A$.

Note that we cannot obtain a $\Sigma_{2}^{1}$ norm whose initial segments are uniformly Borel. This would imply the existence of an uncountable sequence of distinct Borel sets of bounded rank, but it is known that this does not follow from ZF [4, Theorem 4.5].

In order to describe the supremum of the ordinals appearing as the halting time of some OTM program, let $\delta_{2}^{1}$ denote the supremum of lengths of $\Delta_{2}^{1}$ wellorders on sets of natural numbers. Let $\delta_{2}^{1}(x)$ denote the supremum of the length of $\Delta_{2}^{1}$ wellorders in the parameter $x$ on sets of natural numbers. Note that a real $x$ is $\Delta_{2}^{1}$ if and only if $\{x\}$ is $\Delta_{2}^{1}$ or even just $\Sigma_{2}^{1}$.

\footnotetext{
${ }^{1}$ If the algorithm terminates in stage $\beta$, the machine halts after at most $\left(\omega^{\omega} \cdot \beta^{\omega}\right) \cdot \beta$ many steps.
} 
Corollary 2.13. The supremum of halting times of OTMs with input $x$ is $\delta_{2}^{1}(x)$.

Proof. Suppose $y$ codes a $\Delta_{2}^{1}$ wellorder in the parameter $x$ of type $\gamma$. Since $y$ is OTM computable, we consider the algorithm which searches through the well-order given by $y$. The algorithm halts at a time at least $\gamma$.

Conversely, let us consider the $\Pi_{1}^{1}$ set in the parameter $x$ of pairs $(y, z)$ such that $y$ codes a wellorder $w$ with a maximal element $l$ and domain the natural numbers and $z$ codes a halting computation along $w$ on input $x$ which halts at $l$. This set contains a $\Pi_{1}^{1}$ singleton $(y, z)$ in the parameter $x$ by Corollary 2.8. Then $y$ codes a $\Delta_{2}^{1}$ wellorder in the parameter $x$ whose order type is the length of the computation.

\section{Tree Representations from Computations}

In this section, we construct a tree representation for an OTM semi-decidable set of reals from finite fragments of OTM computations. The tape content over an entire halting OTM computation on countable input by a program $P$ can be viewed as an $\omega_{1} \times \omega_{1}$ matrix filled with zeroes and ones. Every row represents the tape content at a given time. If we add a state and a head position per row, the computation is entirely captured in the resulting diagram:

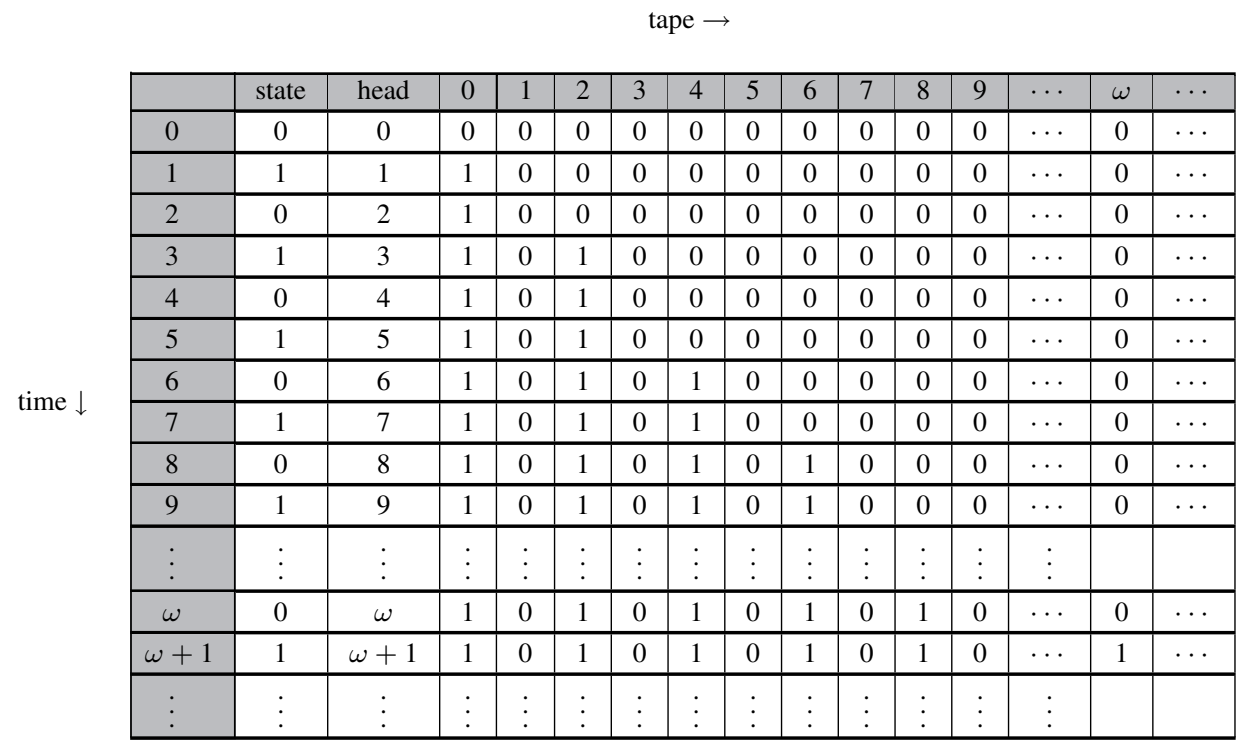

We will approximate similar diagrams by adding single bits of information. A tape bit $(\alpha, \beta, c, \lambda)$ will consist of:

(1) a coordinate $(\alpha, \beta)$ in the $\omega_{1} \times \omega_{1}$ matrix representing time $\alpha$ and tape cell $\beta$

(2) the cell content $c \in\{0,1\}$

(3) a countable limit ordinal (or zero) $\lambda$ - this number will be used to control the limit behavior.

Per row we also need a machine bit $[\alpha, s, \gamma, \lambda]$ containing the following information:

(1) some time $\alpha$, corresponding to the row in the matrix

(2) a machine state $s$ of $P$

(3) a head position $\gamma \in \omega_{1}$

(4) a countable limit ordinal (or zero) $\lambda$ - this number will be used to control the limit behavior.

We will use the symbol ' $\cdot$ ' if we do not want to specify a certain component of a tape or machine bit in the argument at hand (i.e. $(0, n, c, \cdot))$. A finite set of tape and machine bits can be coded into a countable ordinal; fix such a coding. We will now define the tree $T$, depending on the program $P$, on $\omega \times \omega_{1}$ : A pair $(t, u) \in{ }^{k} \omega \times{ }^{k} \omega_{1}$ is in $T$ if and only if: 
(1) The set coded by $u_{j}$ contains the bits coded by $u_{i}$ for $0 \leq i \leq j<n$.

(2) Every $u_{i}$ contains at most one machine bit for each $\alpha$ and at most one tape bit for every pair of $\alpha$ and $\beta$.

(3) For every tape bit $(0, n, c, \cdot)$ of $u_{i}$ with $n<k$, we have that $t=s_{k}$, i.e. $t$ serves as the initial segment of the initial tape contents of the partial computation.

(4) $u_{0}$ contains a machine bit of the form $[0,0, \cdot, \cdot]$ and a tape bit of the form $(0,0,0, \cdot)$. Also it contains a machine bit $[\alpha, s, \gamma, \cdot]$ plus a tape bit $(\alpha, \gamma, c, \cdot)$ where $P$ does not contain an instruction for machine state $s$ and currently read symbol $c$, i.e. $\alpha$ is a halting time. So the beginning and the end of the partial computation are fixed.

(5) As soon as we have information about a tape cell at time $\alpha$, we also know the machine state and head position: If $u_{i}$ contains a tape bit $(\alpha, \cdot, \cdot, \cdot)$, it also contains a machine bit $[\alpha, \cdot, \cdot, \cdot]$.

(6) We always know the tape cell to be read by the read-write head: If $u_{i}$ contains a machine bit $[\alpha, \cdot, \gamma, \cdot]$, it contains a tape bit $(\alpha, \gamma, \cdot, \cdot)$.

(7) If $u_{i}$ contains a tape bit $(\alpha, \beta, c, \cdot), u_{i+1}$ contains bits immediately above (only if $\alpha$ is a successor ordinal) and below along the time axis: Let $[\alpha, s, \gamma, \cdot]$ be the corresponding machine bit given by rule 5 . If $\beta=\gamma$ we require $u_{i+1}$ to contain a tape bit $(\alpha+1, \gamma, \cdot, \cdot)$ and a machine bit $[\alpha+1, \cdot, \cdot, \cdot]$ as required by the program $P$. If $\alpha$ is a successor, we also similarly require the tape and machine bit of the form $[\alpha-1, \cdot, \cdot, \cdot]$ that $P$ implies. Except for those tape bits, all the other tape cells should not change their content, so we add tape bits $(\alpha+1, \beta, c, \cdot)$ if $\beta \neq \gamma$. Again, if $\alpha$ is a successor, we add such tape bits $(\alpha-1, \beta, c, \cdot)$ for all $\beta$ but the one for which we already added such a bit according to $P$.

(8) For tape bits of limit times we have to ensure that the tape contents are inferior limits over earlier times: If $\lambda$ is a limit ordinal, and $(\lambda, \beta, c, \cdot)$ is a tape bit of $u_{i}$. Suppose $c=0$. Then there is a tape bit $(\alpha, \beta, 0, \cdot)$ with $\alpha<\lambda$ in $u_{i+1}$ and $\alpha>\alpha^{\prime}$ for all bits $\left(\alpha^{\prime}, \beta, \cdot, \cdot\right)$ in $u_{i}$ with $\alpha^{\prime}<\lambda$. If $c=1$ then there is a tape bit $(\alpha, \beta, 1, \lambda)$ in $u_{i+1}$ with $\alpha<\lambda$ and where $\alpha$ is larger than any time of a similar bit in $u_{i}$. Let $\alpha^{\prime}$ be minimal such that $u_{i+1}$ contains a tape bit of the form $\left(\alpha^{\prime}, \beta, 1, \lambda\right)$. Then every tape bit for tape cell $\beta$ and time $\bar{\alpha}$ between $\alpha$ and $\lambda$ in $u_{i+1}$ must be of the form $(\bar{\alpha}, \beta, 1, \cdot)$.

(9) We also want the machine state at limit times to be a lim inf: If $\lambda$ is a limit ordinal and $u_{i}$ contains a machine bit $[\lambda, s, \cdot, \cdot], u_{i+1}$ contains a machine bit $[\alpha, s, \cdot, \lambda]$ where $\alpha<\lambda$ and where $\alpha$ is larger than any time of a similar bit in $u_{i}$. Let $\alpha^{\prime}$ be minimal such that $u_{i+1}$ contains a machine bit of the form $\left[\alpha^{\prime}, s, \cdot, \lambda\right]$. Then every machine bit for time $\bar{\alpha}$ between $\alpha$ and $\lambda$ in $u_{i+1}$ must be of the form $\left[\bar{\alpha}, s^{\prime}, \cdot, \cdot\right]$ where $s^{\prime} \geq s$.

(10) Finally, we want to make the head position at limit times a liminf as in the definition of OTMs. If $\lambda$ is a limit ordinal, then for every machine bit $[\lambda, s, \gamma, \cdot]$ of $u_{i}$ one of the following conditions hold: Either there is a machine bit $[\alpha, s, \gamma, \lambda]$ in $u_{i+1}$ with $\alpha<\lambda$ where $\alpha$ is larger than any time of a similar bit in $u_{i}$ and for every machine bit in $u_{i+1}$ of the form $\left[\alpha^{\prime}, s, \gamma^{\prime}, \cdot\right]$ where $\alpha^{\prime}$ is between $\alpha$ and $\gamma$ we have $\gamma^{\prime} \geq \gamma$. Or, alternatively, $u_{i+1}$ does not contain a bit of the form $[\alpha, s, \gamma, \lambda]$, then we require that there is a bit $\left[\alpha, s, \gamma^{\prime}, \lambda\right]$ in $u_{i+1}$ that is not in $u_{i}$ where $\gamma^{\prime}<\gamma$ and $\gamma^{\prime}$ is greater or equal to any $\gamma^{\prime \prime}<\gamma$ in any bit of $u_{i+1}$.

This means that every entry of the matrix given by $u_{i}$ is extended both up- and downwards along the time axis in $u_{i+1}$ while respecting the behavior of the program $P$ and the limit rules involved in the definition of OTMs.

Let us, in the following, write $\operatorname{dom}\left(u_{i}\right)$ for the set of $\alpha$ such that $u_{i}$ contains a bit of the form $(\alpha, \cdot, \cdot, \cdot)$. Moreover, let $\operatorname{dom}(u)=\bigcup_{i \in \omega} \operatorname{dom}\left(u_{i}\right)$.

\section{Lemma 3.1. $T$ projects to the set of reals semi-decided by $P$.}

Proof. First let $x$ be semi-decidable by $P$, i.e. $P(x) \downarrow$. We will show how to use the halting computation $C$ to find a branch of $T_{x}$. Let $\left(\lambda_{i}\right)_{i \in \omega}$ be an enumeration of the limit times involved in $C$. Let $\left[\lambda_{i}, s_{i}, \gamma_{i}, \cdot\right]$ be the corresponding machine bits, and $\left(\lambda_{i}, \gamma_{i}, c_{i}, \cdot\right)$ the corresponding tape bits according to $C$, for $i \in \omega$. We can make sure that $u_{i}$ contains both $\left[\lambda_{i}, s_{i}, \gamma_{i}, \cdot\right]$ and $\left(\lambda_{i}, \gamma_{i}, c_{i}, \cdot\right)$ and tape and machine bits $[\alpha, \cdot, \gamma, \cdot],(\alpha, \gamma, \cdot, \cdot)$ with $\lambda_{m}<\alpha<\lambda_{n}$ for any $m<n<i$. Let us close $\left(u_{i}\right)_{i<\omega}$ under above rules using bits compatible with $C$. It is clear that for any two consecutive limits $\lambda_{k}$ and $\lambda_{l}$, there is some $u_{i}$ which contains bits $(\alpha, \cdot, \gamma, \cdot),[\alpha, \gamma, \cdot, \cdot]$ with $\lambda_{k}<\alpha<\lambda_{l}$. Since all bits are chosen from $C$, the gaps between the $\lambda_{i}$ can be filled and $\left(u_{i}\right)_{i<\omega}$ forms a branch in $T_{x}$. 
Now let $\left(u_{i}\right)_{i \in \omega}$ be a branch of $T_{x}$. We need to prove that the computation $C$ by $P$ on input $x$ halts. Let $\left(\lambda_{i}\right)_{i \in \omega}$ be an enumeration of the limits in $\operatorname{dom}(u)$.

Claim. The ordinals in $\operatorname{dom}(u)$ are exactly the ordinals $\lambda_{j}+n$ for $j, n \in \omega$.

Proof of Claim. By the rules above it is clear that every ordinal of the form $\lambda_{j}+n$ is in $\operatorname{dom}(u)$. Suppose that $\mu$ is a limit and $\mu+n \in \operatorname{dom}\left(u_{i}\right)$ where $\mu \neq \lambda_{j}$ for all $j \in \omega$. Then it follows from the rules that $\mu \in \operatorname{dom}\left(u_{i+n}\right)$, a contradiction.

The set of bits in $\left(u_{i}\right)_{i \in \omega}$ induce a partial matrix $U$ of the type pictured above. We call a submatrix according to $P$, if the machine state, head position, and tape contents change only as dictated by $P$.

Claim. For $\lambda \in\left(\lambda_{i}\right)_{i \in \omega}$ the submatrix of $U$ induced by the rows $\lambda+n$ for all $n \in \omega$ is according to $P$.

Proof of Claim. Let $n \in \omega$ and choose $i$ minimal such that $\exists m \lambda+m \in \operatorname{dom}\left(u_{i}\right)$. The rules dictate that, for any $m \in \omega, u_{i+|n-m|}$ contains unique machine bits for all rows between $\lambda+m$ and $\lambda+n$. Those machine bits and also the tape contents covered by bits present in $u_{i}$ are changed only according to $P$. Of course, new tape cells might have been introduced by tape bits in $u_{j}, j>i$. But for any such given tape cell $\beta$, its content is kept constant except for actions of $P$. If at any stage a new bit would have been required to be added that conflicts with bits already present in $u$, the branch would not have been extended further.

It remains to show that at limit times, machine state, head positions, and tape contents are inferior limits.

Claim. Let $\lambda$ be in $\left(\lambda_{i}\right)_{i \in \omega}$. Let $\left(\alpha_{j}\right)_{j<\nu}$ be an increasing enumeration of $\operatorname{dom}(u) \cap \lambda$. Then:

(i) For every tape bit $(\lambda, \beta, c, \cdot), c$ is the liminf over the $d$ in tape bits of the form $\left(\alpha_{j}, \beta, d, \cdot\right)$ in $\bigcup_{i \in \omega} u_{i}$.

(ii) For every machine bit $[\lambda, s, \cdot, \cdot], s$ is the liminf over the $r$ in machine bits of the form $\left[\alpha_{j}, r, \cdot, \cdot\right]$ in $\bigcup_{i \in \omega} u_{i}$.

(iii) For every machine bit $[\lambda, s, \gamma, \cdot], \gamma$ is the liminf over the $\delta$ in machine bits of the form $\left[\alpha_{j}, s, \delta, \cdot\right]$ in $\bigcup_{i \in \omega} u_{i}$, if this liminf is a head position occurring in $\bigcup_{i \in \omega} u_{i}$, or $\gamma$ is the least head position occurring in $\bigcup_{i \in \omega} u_{i}$ that is greater than the liminf.

\section{Proof of Claim.}

(i) Choose $u_{i}$ such that $(\lambda, \beta, c, \cdot)$ is in $u_{i}$. Let $\left(\left(\alpha_{k}, \beta, d_{k}, \cdot\right)\right)_{k \in \mu}$ be an increasing (in $\left.\alpha_{k}\right)$ enumeration of the tape bits in $\left(u_{j}\right)_{i<j<\omega}$ where $\alpha_{j}<\lambda$. First consider $c=0$. The rules imply that $\left(d_{k}\right)_{k \in \mu}$ contains an unbounded sequence of $0 \mathrm{~s}$, hence $c$ is in fact the inferior limit. Now suppose $c=1$. In $u_{i+1}$ a tape bit of the form $(\alpha, \beta, 1, \lambda)$ is added and all $d_{k}$ where $\alpha_{k}>\alpha$ are $\geq 1$.

(ii) Choose $u_{i}$ such that $[\lambda, s, \cdot, \cdot]$ is in $u_{i}$. Let $\left(\left[\alpha_{k}, s_{k}, \cdot, \cdot\right]\right)_{k \in \mu}$ be an increasing (in $\left.\alpha_{k}\right)$ enumeration of the machine bits in $\left(u_{j}\right)_{i<j<\omega}$ where $\alpha_{j}<\lambda$. In $u_{i+1}$ a machine bit of the form $[\alpha, s, \cdot, \lambda]$ is added, where $\alpha$ is greater than any time of a similar bit in $u_{i+1}$. Indeed in every $u_{j}$ where $j>i$ such a bit is added, so $\left(s_{k}\right)_{k<\mu}$ contains $s$ unboundedly often. Also, the rules imply that every $s_{k} \geq s$ for for all $\alpha_{k} \geq \alpha$.

(iii) Choose $u_{i}$ such that $[\lambda, s, \gamma, \cdot]$ is in $u_{i}$. Let $\left(\left[\alpha_{k}, s, \gamma_{k}, \cdot\right]\right)_{k \in \mu}$ be an increasing (in $\alpha_{k}$ ) enumeration of the machine bits in $\left(u_{j}\right)_{i<j<\omega}$ where $\alpha_{j}<\lambda$ (note that we only consider bits with machine state $s$ ).

Case 1. In $u_{i+1}$ a machine bit of the form $[\alpha, s, \gamma, \lambda]$ is added, where $\alpha$ is greater than any time of a similar bit in $u_{i+1}$. Indeed in every $u_{j}$ where $j>i$ such a bit is added, so $\left(\gamma_{k}\right)_{k<\mu}$ contains $\gamma$ unboundedly often. Also, the rules imply that every $\gamma_{k} \geq \gamma$ for for all $\alpha_{k} \geq \alpha$.

Case 2. No such bit is added in any $u_{j}, i<j$. Then by the rules, $\left(\gamma_{k}\right)_{k \in \mu}$ is strictly increasing below $\gamma$. Note that by the rules there is no head position in $u$ that is between $\sup _{k \in \mu}\left(\gamma_{k}\right)$ and $\gamma$. So even if $\lim \inf _{k<\mu}\left(\gamma_{k}\right)<\gamma$, the partial computation behaves as if $\gamma$ was indeed the lim inf.

We can alter rule 1 in the definition of the tree of partial computations to have the nodes $u_{i}$ contain information about when which bits where added, allowing $u_{i}$ to be decoded into $\left(u_{j}\right)_{j<i}$. Let us assume this extra requirement for the next lemma. We consider the lexicographical well-order $<_{l e x}$ between bits. If $d=\left\{d_{i}: i \leq m\right\}$ and $e=\left\{e_{i}: i \leq n\right\}$ 
are finite sets of bits with $d_{0}<_{\text {lex }} \ldots<_{\text {lex }} d_{m}$ and $e_{0}<_{\text {lex }} \ldots<_{\text {lex }} e_{n}$, we define $d<_{\text {lex }} e$ if $|d|<|e|$, or $|d|=|e|$ and $d_{i}<_{l e x} e_{i}$ for the least $i$ with $d_{i} \neq e_{i}$. If $u, v$ both satisfy the properties of the sequence $u$ in the definition of $T$, we can decode sequences $u_{0}, u_{1}, \ldots, u_{m}=u$ and $v_{0}, v_{1}, \ldots, v_{n}=v$ from $u$ and $v$ such that for all $i<m, u_{i}$ extends to $u_{i+1}$ by a set of additional bits, which we call $u_{i+1}^{+}$, as stated by the rules for $T$, and similarly $v_{j}$ for $j<n$. Let us define $u<_{\text {tree }} v$ by $m<n$, or $m=n$ and $u_{i}^{+}<_{l e x} v_{i}^{+}$for the least $i$ with $u_{i} \neq v_{i}$.

Lemma 3.2. $T$ has pointwise leftmost branches with respect to $<_{\text {tree, }}$ i.e. that for every input $x$ on which the computation halts, the tree $T_{x}$ has a branch $b$ so that $b_{n} \leq_{\text {tree }} c_{n}$ for every branch $c$ of $T_{x}$ and for every $n$.

Proof. Let us consider the computation with input $x$. Let $b_{0}=\{[0,0,0,0],(0,0,0,0),[\alpha, s, \gamma, 0],(\alpha, \gamma, c, 0)\}$, where $\alpha$ is the halting time, $s$ is the machine state at time $\alpha, \gamma$ is the head position at time $\alpha$, and $c$ is the content of cell $\gamma$ at time $\alpha$. Let $b_{n+1}^{+}$be $\leq_{l e x}$-least such that the extension $b_{n+1}$ of $b_{n}$ by $b_{n+1}^{+}$describes a fragment of the given computation and is in $T$. Suppose towards a contradiction that $c$ is a branch in $T_{x}$ and $n$ is minimal with $c_{n}<_{\text {tree }} b_{n}$. We can recover the predecessors $b_{0}, \ldots, b_{n-1}$ of $b_{n}$ and $c_{0}, \ldots, c_{n-1}$ of $c_{n}$. If $b_{i}<_{\text {tree }} c_{i}$ for some $i<n$, then $b_{n}<_{\text {tree }} c_{n}$, contradicting the choice of $n$. Hence $b_{i}=c_{i}$ for all $i<n$ by minimality of $n$. This implies $b_{n}^{+} \leq_{\text {lex }} c_{n}^{+}$by the definition of $b$ and thus $b_{n} \leq_{\text {tree }} c_{n}$, contradicting the assumption.

Remark 3.3. A stricter variation of $T$ would only allow extensions by a minimal number of bits necessary to fulfill the conditions, and require the bits to be chosen $<_{l e x}$-minimal in the sense that an extension of a node may not add $<_{\text {lex }}$-smaller bits which satisfy the same requirement as a given bit. If we consider this variation and change the definition of $T$ so that $u_{i}$ consists only of the additional bits relative to $\bigcup_{j<i} u_{j}$, it is not hard to see that $T$ has pointwise leftmost branches with respect to $<_{l e x}$.

Remark 3.4. The tree $T$ induces a $\Sigma_{2}^{1}$ scale on the set of reals semi-decided by $P$. Let $x \leq_{n} y$ if both $P(x)$ and $P(y)$ halt and $b_{x}(n) \leq_{\text {tree }} b_{y}(n)$, for the leftmost branches $b_{x} \in T_{x}$ and $b_{y} \in T_{y}$, respectively, or $P(x)$ halts and $P(y)$ does not halt. The relation $x<_{n} y$ has an analogous definition with $\leq_{\text {tree }}$ replaced by $<_{\text {tree }}$. To prove that $T$ is the tree from a scale, it is sufficient to show that the relations $\leq_{n}$ and $<_{n}$ induced by $T$ are OTM semi-decidable. We semi-decide $x \leq_{n} y$ by simulating $P$ on the inputs $x$ and $y$, as in Corollary 2.12. If $P(x)$ halts before $P(y)$, we halt the program. If $P(x), P(y)$ halt at the same step, we run an OTM computation to determine whether $b_{x}(n) \leq_{n} b_{y}(n)$ and halt the program, if this is the case. Otherwise we let the program diverge. The argument for $<_{n}$ is similar.

Remark 3.5. As a natural extension of the scale property, we might ask for a tree $T$ projecting to a $\Sigma_{2}^{1}$ universal set $A$ such that $T_{x}$ has a unique infinite branch for every $x \in A$. Let us argue that the existence of such a tree is not provable in ZF. Assuming such a tree $T$ exists, let $S=\left\{\left(s,\left(\left(s_{0}, t_{0}\right), \ldots,\left(s_{n-1}, t_{n-1}\right)\right)\right): n \in \omega,(s, t) \in T\right\}$. Then $A=p[T]=p[S]$ and there is a unique $b_{x} \in S_{x}$ for every $x \in A$, and $b_{x} \neq b_{y}$ for all $x \neq y$. Since for each $\alpha<\omega_{1}$ the projection of $S$ restricted to ordinals below $\alpha$ is an injective image of a closed set and hence Borel, there is an $n$ such that the set $B$ of values of $b_{x}(n)$ for $x \in A$ is unbounded in $\omega_{1}$. Let us choose the leftmost branch $\left(x_{\alpha}, b_{\alpha}\right)$ in $S$ with $b_{\alpha}(n)=\alpha$ for each $\alpha \in B$. We have defined an uncountable sequence $\left(x_{\alpha}: \alpha \in B\right)$ of distinct reals. However, there is no such sequence in the symmetric model for the Levy collapse $\operatorname{Col}\left(\omega,<\aleph_{\omega}\right)$, as was pointed out to the authors by Daisuke Ikegami.

It is possible to prove Shoenfield absoluteness without referring to tree representations from descriptive set theory, using only computational means:

Remark 3.6. The tree of partial computations may be used instead of the Shoenfield tree to show that every $\Sigma_{2}^{1}$ set is OTM semi-decidable. We can see that $\Sigma_{1}^{1}(x)$ sets are ordinal semi-decidable via a depth-first search for a witness for the $\Sigma_{1}^{1}(x)$ statement. The length of this search is bounded by the least $\alpha$ such that $L_{\alpha}[x]$ is a model of KP and $\Sigma_{1}$-separation (see [1, Theorem 9.6]), and this ordinal $\alpha$ is computable on input $x$ by recursively writing codes for $L_{\beta}[x]$ for increasing $\beta$ while checking the axioms. Hence $\Sigma_{1}^{1}$ and $\Pi_{1}^{1}$ sets are OTM decidable. To semi-decide a $\Sigma_{2}^{1}$ set $A=p[B]$ with $B$ in $\Pi_{1}^{1}$, we now search on input $x$ for a real $y$ and a branch in the tree of partial computations for $B$ on input $(x, y)$. 


\section{Applications}

In [7, Definition 1] the programs that steer the computations of OTMs are defined with the following condition: If the machine is currently in state $s$ and the machine's read-write head currently reads symbol $c$, then the program contains at most one command for that situation. This way, when Koepke defines the ordinal computation by a program $P$, he can refer to the unique command in a given situation. Instead, for the present section, we shall drop the above restriction on programs and define ordinal computations in a way that, in successor steps, the lexicographically least instruction (if there is one that suits the current situation) is chosen to determine the next machine step. This allows us to define non-deterministic ordinal computations as follows.

Definition 4.1. Given program $P$ and an input (i.e. an initial tape configuration), the non-deterministic ordinal Turing computation (NOTM computation) by $P$ is defined like the ordinal computation by $P$ ([7, Definition 2]), except that in successor steps any suitable command may define the machine's next step.

NOTM computations may be used to define sets of reals.

Definition 4.2. A set of reals $A \subseteq{ }^{\omega} \omega$ is NOTM semi-decidable if there is a program $P$ such that

$$
x \in A \leftrightarrow \text { there is a halting NOTM computation by } P \text { on input } x
$$

Consider a countable substructure of a transitive set containing such a computation as an element. Then the image of the computation under the collapsing map is a countable halting NOTM computation by $P$ on input $x$.

As in the case of classical Turing decidability, given a coding of the "choices" that a NOTM computation makes, NOTM decidability can be verified deterministically:

Lemma 4.3. There is a program $Q$ such that for every program $P$ and every real input $x$, there is a real $z$ such that the OTM computation by $Q$ on inputs $P, x$, and $z$ halts if and only if some NOTM computation by $P$ on input $x$ halts.

Proof. Let us define $z$ to code two reals $z_{1}$ and $z_{2}$. Let $z_{1}$ code a well-order on $\omega$ of order type the (countable) length of the NOTM computation by $P$ on input $x$. Let $z_{2}$ be such that in machine step otp $z_{z_{1}}(i)$, the OTM computation by $P$ on input $x$ selects the $z_{2}(i)$-th least command $P$ contains for that situation. Note that both otp $z_{z_{1}}$ and otp $z_{z_{1}}^{-1}$ are OTM computable functions. Now the program $Q$ is essentially a universal OTM which selects the $z_{2}(i)$-th command in $P$ in the $\operatorname{otp}_{z_{1}}(i)$-th simulation step.

This settles the question of whether NOTMs compute more sets of reals than OTMs:

Proposition 4.4. Every NOTM (semi-)decidable set of reals is already OTM (semi-)decidable.

Proof. Let $A \subseteq{ }^{\omega} \omega$ and suppose that $Q$ is the program from Lemma 4.3. Then $A$ is NOTM semi-decidable if and only if there is a program $P$ so that for every input $x$ there is a real $z$ such that the OTM computation by $Q$ on inputs $P, x$, and $z$ halts. Since this is a $\Sigma_{2}^{1}$ statement, $A$ is $\Sigma_{2}^{1}$ and hence OTM semi-decidable.

Note now that the existence of certificates $z$ established in Lemma 4.3 is absolute. Thus, if there are any certificates, there is one in $L$. Using Shoenfield absoluteness, we could search for such a $z$ through $L$, using the OTM computable recursive truth predicate from [7]. Instead, let us search for a certificate via the tree of partial computations:

Lemma 4.5. Given a program $P$ and an element $(s, u)$ of the full tree on $\omega \times \omega_{1}$, we can OTM decide the question of whether or not $(s, u)$ is an element of the tree of partial computations according to $P$.

Proof. We first have the OTM check whether $u$ codes a set of tape and machine bits. If yes, we can easily check the finitely many conditions (rules 1-10) if $u$ is a partial computation by $P$ on some input that is compatible with $s$. 
With the preceding lemma, we can use a variant of Algorithm 2.3 to find branches in the tree of partial computations. Since Propositions 1 and 2 hold also for our algorithm operating on the tree of partial computations, we get:

Proposition 4.6. There is an algorithm such that, if $A$ is NOTM semi-decidable by the program $P$, then, given $x$ as an input, the algorithm will find a real $z \in{ }^{\omega} \omega$ such that the OTM computation by $Q$ (cf. Lemma 4.3) on inputs $P, x$, and $z$ halts if $x \in A$ and diverges otherwise.

Proof. If $x$ is in $A$, there is a $z$ in $L$ such that the OTM computation by $Q$ on inputs $P, x$, and $z$ halts. An argument analogous to Propositions 2.2 and 2.4 shows that given a real $x$, a straighforward adaptation of algorithm 2.3 will find a branch of the form $(x, c)$ in the tree $T$ of partial computations by $P$, if any exists. From $c$ the desired $z$ can be easily decoded.

The tree representation allows us to generalize the results in Section 2 to sets of reals semi-decided by ordinal machines with upper bounds on the halting times.

Definition 4.7. Suppose $f$ is a function from the reals to the ordinals. Let us say that a set of reals $A$ is $f$-semidecidable or $\Gamma_{f}$ if there is a OTM program $P$ semi-deciding $A$ such that $P$ halts before time $f(x)$ on input $x$ if it halts at all.

For our purpose, we are interested in functions of the following form:

Definition 4.8. Suppose $f$ is a function from the reals to the ordinals. We call $f$ multiplicatively closed if $f(x) \leq$ $f(\langle x, y\rangle)$ for all reals $x$ and $y$ and $f(x)$ as an ordinal is closed under ordinal multiplication. Let us call $f$ admissible if furthermore $f(x)$ is $x$-admissible for all reals $x$.

The classes $\Gamma_{f}$ for admissible $f$ with values strictly above $\omega$ range from $\Pi_{1}^{1}$ to $\Sigma_{2}^{1}$. Recall the definition of $\delta_{2}^{1}$ from the paragraph before Corollary 2.13.

Lemma 4.9. Let $f(x)=\omega_{1}^{x}$ and $g(x)=\delta_{2}^{1}(x)$. Then $\Gamma_{f}$ is the class of $\Pi_{1}^{1}$ sets and $\Gamma_{g}$ is the class of $\Sigma_{2}^{1}$ sets.

Proof. Suppose that $A$ is $f$-semi-decidable via $P$ and $x$ is a real. Then $x \in A$ if and only if in every countable model of KP every computation by $P$ with input $x$ halts. Since such models can be coded into reals, $A$ is $\Pi_{1}^{1}$. Suppose $A$ is $\Pi_{1}^{1}$ and $x \in A$ if and only if $T_{x}$ is wellfounded. Then $\operatorname{rank}\left(T_{x}\right)<\omega_{1}^{x}$ and hence the algorithm searching for a branch in the Shoenfield tree halts before $\omega_{1}^{x}$. The statement for $\Sigma_{2}^{1}$ sets follows from Corollary 2.13.

Corollary 4.10. Suppose $f$ is multiplicatively closed. Then every $\Gamma_{f}$ set has a $\Gamma_{f}$ norm.

Proof. Suppose a set in $\Gamma_{f}$ is semi-decidable by a program $P$ with halting time bounded by $f$. Let $\phi(x)$ be the halting time of $P$ on input $x$. Since $f$ is multiplicatively closed, $\phi$ is a $\Gamma_{f}$-norm as in the proof of Corollary 2.12.

Corollary 4.11. Suppose $f$ is admissible. Then every $\Gamma_{f}$ binary relation has a uniformization with graph in $\Gamma_{f}$.

Proof. Suppose a relation in $\Gamma_{f}$ is semi-decidable by a program $P$ with halting time bounded by $f$. We apply the algorithm for searching through the Shoenfield tree to the tree of partial computations. Let us consider a program $R$ for the variant of Algorithm 2.3 which on input $(x, y)$ searches for a real $z$ and a branch in the tree of halting computations of $P$ with input $(x, z)$. We claim that if the program finds such a pair, then this happens before the time $\alpha=f(\langle x, z\rangle)$. Let us assume towards a contradiction that $z$ and the corresponding computation of $P$ are found at a time $\gamma \geq \alpha$. If $\gamma=\alpha$, we map each $n \in \omega$ to the time at which $z\lceil n$ appears first in the search, and thus obtain a $\Sigma_{1}^{L_{\alpha}[x, y]}$ definable cofinal map $h: \omega \rightarrow \alpha$. If $\gamma>\alpha$, there is a $t \in{ }^{<\omega} \omega$ which appears first at the time $\alpha$, and we obtain a $\Sigma_{1}^{L_{\alpha}[x, y]}$ definable cofinal map $h:\left\{s \in{ }^{<\omega} \omega: s<_{\text {lex }} t\right\} \rightarrow \alpha$ by mapping $s$ to the time at which it first appears in the search. This contradicts the $\langle x, z\rangle$-admissibility of $\alpha$.

At this point, we let $R$ halt if $y=z$ and let $R$ diverge otherwise. For any real $x$ in the domain of the relation, let $(g(x), b(x))$ be $\leq_{l e x}$-least such that $b(x)$ is a branch in the tree of partial computations with input $(x, g(x))$. Then $R(x, g(x))$ halts and $R(x, z)$ diverges for all $z \neq g(x)$. Hence the graph of $g$ is in $\Gamma_{f}$. 
Let us now consider ordinal machines with a set of reals as oracle as in [3]. In a query state in a computation, the program asks whether the sequence on the initial segment of length $\omega$ of the tape is an element of the set. Let us write $P^{A}(x)$ for the OTM computation by the program $P$ with oracle $A$ on input $x$. Let us also fix a computable enumeration $\left(P_{n} \mid n \in \omega\right)$ of all programs.

Definition 4.12. The halting problem relative to a set of reals $A$ or jump of $A$ is defined as $A^{\boldsymbol{\nabla}}=\{(n, x) \mid$ $\left.P_{n}^{A}(x) \downarrow\right\}$.

The halting problem 0 is a $\Sigma_{2}^{1}$ set, in fact we have:

Proposition 4.13. The halting problem $0^{\nabla}$ is $\Sigma_{2}^{1}$ universal. If $n \geq 1$ and $V=L$, then the $n^{\text {th }}$ iterated jump $0^{\nabla}$ is $\Sigma_{n+1}^{1}$ universal.

Proof. Every halting computation with countable input halts at a countable time (see Remark 2.5). Hence $(m, x) \in$ $0^{\mathbf{\nabla}}$ is described by a $\Sigma_{n+1}^{1}$ formula stating the existence of a wellorder $w$ on the natural numbers with largest element $l$ together with a sequence indexed by $w$, coding a computation of $P_{m}$ with input $x$ and oracle $0^{\mathbf{v}(n-1)}$ halting at $l$. Let us suppose that $A$ is defined by the formula $\exists x \varphi(x, y)$, where $\varphi$ is $\Pi_{n}^{1}$. We consider a program searching through $L$ for a witness for $\varphi$ as in [7], using the oracle $0^{\mathbf{v}(n-1)}$ to verify $\varphi(x)$ for reals $x$. This program identifies $A$ as a section of $0^{\mathbf{\nabla}}$.

In particular, the $\Sigma_{n+1}^{1}$ sets are exactly the OTM semi-decidable sets in a $\Sigma_{n}^{1}$ oracle for $n \geq 1$, if $V=L$. Let us show that this remains true when $\kappa \geq \omega_{1}$ many Cohen reals are added to $L$ by the forcing $\operatorname{Add}(\omega, \kappa)$.

Lemma 4.14. Suppose that $V=L[G]$, where $G$ is $A d d(\omega, \kappa)$-generic over $L$, and $\kappa \geq \omega_{1}$. Then $0^{n}$ is $\Sigma_{n+1}^{1}$ universal for all $n \geq 1$.

Proof. Suppose that $x$ is a real in $L[G]$. There are an $A d d(\omega, 1)$-generic filter $g_{0}$ with $L[x]=L\left[g_{0}\right]$ and an Add $(\omega, \kappa)$-generic filter $g_{1}$ over $L\left[g_{0}\right]$ with $L[G]=L\left[g_{0}\right]\left[g_{1}\right]$. Let us consider the case $n=2$ and suppose $\varphi$ is a binary $\Pi_{2}^{1}$-formula. Then $\exists y \varphi(x, y)$ holds in $L[G]$ if and only if $\exists \sigma \in N \Vdash_{A d d(\omega, 1)} \varphi(y, \sigma)$ holds in $L[x]$, where $N$ is the set of nice $A d d(\omega, 1)$-names for reals in $L[x]$. Since nice names for reals are coded by reals, this is a $\Sigma_{3}^{1}$ statement in $L[x]$. We can now express any $\Sigma_{n+1}^{1}$ statement about $x$ in $L[G]$ by a $\Sigma_{n+1}^{1}$ statement in $L[x]$ uniformly in $x$ for all $n \geq 1$ in a similar fashion. This is proved by induction on $n$. Every such set is a section of $0^{\mathbf{v}}$ by the proof of the previous proposition.

It is also consistent with ZFC that the iterated jumps have a lower complexity. Note that the assumption that $\omega_{1}^{L[x]}<\omega_{1}$ for every real $x$ may be obtained by forcing with the Levy collapse $\operatorname{Col}(\omega,<\kappa)$ below an inaccessible cardinal $\kappa$.

Lemma 4.15. Suppose that $\omega_{1}^{L[x]}<\omega_{1}$ for every real $x$. Then $0^{\mathbf{v} n}$ is a $\Delta_{3}^{1}$ set for all $n \geq 1$.

Proof. Let us consider the $\Pi_{2}^{1}$ set $A$ of pairs $(x, y)$ such that $y$ codes $L_{\gamma}[x]$ and $\gamma$ is the least $x$-admissible ordinal above $\omega_{1}^{L[x]}$. We can compute the truth value of $x \in 0^{\mathbf{v}}$ in $L_{\gamma}[x]$ using an algorithm which has access to $n$ distinct tapes of length $\omega_{1}^{L[x]}+1$. The original program runs on tape $n$. Whenever the oracle $0^{\mathbf{v}}$ is called on tape $i$ for $1<i \leq n$, the oracle is computed on tape $i-1$ by a subroutine of length $\omega_{1}^{L[x]}+1$. The $\Pi_{2}^{1}$ description of $A$ yields a $\Delta_{3}^{1}$ description of the set of pairs $(x, n)$ with $x \in 0^{\mathbf{v} n}$.

The two previous lemmas imply that the complexity of $0^{\mathbf{v}}$ cannot be computed in ZFC for $n \geq 2$, even if the size of the continuum is known.

\section{Further Questions}

A set of reals $A$ is said to be $\Sigma_{2}^{1}$ in a countable ordinal $\alpha$ if there is a $\Sigma_{2}^{1}$ formula $\varphi(x, y)$ such that for all reals $y$ coding $\alpha$ and all reals $x, x \in A$ if and only if $\varphi(x, y)$ holds, i.e. the $\Sigma_{2}^{1}$ definition is independent of the coding of 
$\alpha$. We leave open whether the sets of reals with a $\Sigma_{2}^{1}$ definition in an ordinal $\alpha$, evaluated in $V^{\operatorname{Col}(\omega, \alpha)}$, are exactly the OTM semi-decidable sets of reals with parameter $\alpha$, and whether sets in these classes can be uniformized by functions with graphs in these classes.

\section{References}

[1] J. Barwise. Admissible Sets and Structures: An Approach to Definability Theory. Springer-Verlag, Berlin, 1975. Perspectives in Mathematical Logic.

[2] G. Hjorth. Vienna notes on effective descriptive set theory and admissible sets. http://www.math.uni-bonn.de/ people/logic/events/young-set-theory-2010/Hjorth.pdf, 2010.

[3] J. D. Hamkins and A. Lewis. Infinite time Turing machines. The Journal of Symbolic Logic, 65(2): 567-604, 2000.

[4] L. Harrington. Analytic determinacy and $0^{\sharp}$. The Journal of Symbolic Logic, 43(4):685-693, 1978.

[5] T. Jech. Set Theory: Springer Monographs in Mathematics, The third millennium edition, revised and expanded. Springer-Verlag, Berlin, 2003.

[6] A. S. Kechris. Classical Descriptive Set Theory, volume 156 of Graduate Texts in Mathematics. SpringerVerlag, New York, 1995.

[7] P. Koepke. Turing computations on ordinals. The Bulletin of Symbolic Logic, 11:377-397, 2005.

[8] P. Koepke. Ordinal computability. In K. Ambos-Spies, B. Löwe, and W. Merkle, editors, Mathematical Theory and Computational Practice, volume 5635 of Lecture Notes in Computer Science, pages 280-289. SpringerVerlag, Berlin, Heidelberg, 2009.

[9] P. Koepke and B. Seyfferth. Ordinal machines and admissible recursion theory, Computation and Logic in the Real World: CiE 2007. Annals of Pure and Applied Logic, 160(3):310-318, 2009. 